\title{
Comments on Finite-time Stability of Time-Delay Systems
}

\author{
Efimov D. ${ }^{1,2,4}$, Polyakov A. ${ }^{1,2}$, Fridman E. ${ }^{3}$, \\ Perruquetti W. ${ }^{1,2}$, Richard J.-P. ${ }^{1,2}$ \\ ${ }^{1}$ Non-A team @ INRIA - LNE, Parc Scientifique de la Haute Borne, 40 avenue Halley, \\ 59650 Villeneuve d'Ascq, France \\ ${ }^{2}$ LAGIS (UMR-CNRS 8146), Ecole Centrale de Lille, BP 48, Cité Scientifique, 59651 \\ Villeneuve-d'Ascq, France \\ ${ }^{3}$ School of Electrical Engineering, Tel-Aviv University, Tel-Aviv 69978, Israel \\ ${ }^{4}$ Department of Control Systems and Informatics, University ITMO, 49 av. Kronverkskiy, \\ 197101 Saint Petersburg, Russia
}

\begin{abstract}
Recently proposed conditions on finite-time stability in time-delay systems are revisited and it is shown that they are incorrect. General comments on possibility of finite-time convergence in time-delay systems and a necessary condition are given.
\end{abstract}

\section{Introduction}

The time-delay dynamical systems attract a lot of attention in different areas of practice Chiasson and Loiseau [3], Erneux [6]. Analysis of stability in these systems is complicated Richard [16], the most of stability conditions deal with linear time-delay models, where the necessary and sufficient conditions have been obtained for some special cases Gu et al. [7], Hale [8], Kolmanovsky and Nosov [11]. Prior to N.N. Krasovskii's papers on Lyapunov functionals and B.S. Razumikhin's papers on Lyapunov functions, L.E. El'sgol'ts (see El'sgol'ts and Norkin [5] and references therein) considered the stability problem of the solution $x(t) \equiv 0$ of time-delay systems by proving that the function $t \rightarrow V(x(t))$ is decreasing. Here $V$ is some Lyapunov function. He showed that it is only possible in some rare special cases. Therefore, there are two generic methods for stability analysis in time-delay systems: Lyapunov-Krasovskii approach and Lyapunov-Razumikhin method. The former one is based on analysis of derivative for a functional, and it provides qualitative and quantitative estimates on 
the system convergence. The latter approach is based on derivative analysis of a function and, from the point of view of the convergence rate, it gives mainly a qualitative conclusion (stability/instability of a time-delay system can be detected without estimation of the convergence rate, see Theorem 1 below).

It is frequently important to quantify the rate of convergence in the system: exponential, asymptotic, finite-time or fixed-time (see the results obtained for ordinary differential equations in Roxin [17], Dorato [4], Moulay and Perruquetti [13], Nersesov et al. [14], Polyakov [15]). Frequently, the homogeneity theory is used to evaluate finite-time or fixed-time stability in the delay-free case Bhat and Bernstein [2], Andrieu et al. [1], Polyakov [15]: for example, if a system is globally attractive and homogeneous of negative degree, then it is finite-time stable. There is a recent interest to analysis of finite-time stability behavior for time-delay systems Karafyllis [9], Moulay et al. [12], Yang and Wang [18, 19]. The paper Karafyllis [9] proposes design of a control, which implicitly contains some prediction mechanisms and time-varying gains in order to compensate the delay influence on the system dynamics and guarantee a kind of finite-time stability for the closed-loop system. The main result of Moulay et al. [12] is given in Proposition 2 below: in order to establish finite-time stability for a functional differential equation it is necessary to find a Lyapunov-Krasovskii functional $V(\phi)$ whose derivative is upper bounded by a certain negative function of the functional $V(\phi)$ itself (it is a more restrictive condition than in the conventional Lyapunov-Krasovskii approach, where a function of $|\phi(0)|$ is required). Yang and Wang $[18,19]$ base their study on the Lyapunov-Razumikhin approach, they claim improvements over earlier results.

In this note, we argue that some key results in Yang and Wang $[18,19]$ are incorrect as stated, and we provide new insight on the features of finite-time stability for time-delay systems. The El'sgol'ts' arguments are recalled.

The outline of this work is as follows. The preliminary definitions and finitetime stability conditions for time-delay systems are given in Section 2. A counterexample to a key result in Yang and Wang $[18,19]$ is presented and discussed in Section 3. A necessary condition for finite-time stability for a class of timedelay systems and some supplementary comments are provided in Section 4 . The result of Moulay et al. [12] is quoted in concluding Section 5. 


\section{Preliminaries}

Consider an autonomous functional differential equation of the retarded type Kolmanovsky and Nosov [11]:

$$
d x(t) / d t=f\left(x_{t}\right), t \geq 0,
$$

where $x \in \mathbb{R}^{n}$ and $x_{t} \in C_{[-\tau, 0]}$ is the state function, $x_{t}(s)=x(t+s),-\tau \leq s \leq 0$ (we denote by $C_{[-\tau, 0]}, 0<\tau<+\infty$ the Banach space of continuous functions $\phi:[-\tau, 0] \rightarrow \mathbb{R}^{n}$ with the uniform norm $\|\phi\|=\sup _{-\tau \leq \varsigma \leq 0}|\phi(\varsigma)|$, where $|\cdot|$ is the standard Euclidean norm); $f: C_{[-\tau, 0]} \rightarrow \mathbb{R}^{n}$ is a continuous function, $f(0)=0$. The representation (1) includes pointwise or distributed retarded systems. We assume that (1) has a solution $x\left(t, x_{0}\right)$ satisfying the initial condition $x_{0} \in$ $C_{[-\tau, 0]}$, which is defined on some finite time interval $[-\tau, T$ ) (we will use the notation $x(t)$ to reference $x\left(t, x_{0}\right)$ if the origin of $x_{0}$ is clear from the context).

The upper right-hand Dini derivative of a locally Lipschitz continuous functional $V: C_{[-\tau, 0]} \rightarrow \mathbb{R}_{+}$along the system (1) solutions is defined as follows for any $\phi \in C_{[-\tau, 0]}$ :

$$
D^{+} V(\phi)=\lim _{h \rightarrow 0^{+}} \sup \frac{1}{h}\left[V\left(\phi_{h}\right)-V(\phi)\right]
$$

where $\phi_{h} \in C_{[-\tau, 0]}$ for $0<h<\tau$ is given by

$$
\phi_{h}= \begin{cases}\phi(\theta+h), & \theta \in[-\tau,-h) \\ \phi(0)+f(\phi)(\theta+h), & \theta \in[-h, 0] .\end{cases}
$$

For a locally Lipschitz continuous function $V: \mathbb{R}^{n} \rightarrow \mathbb{R}_{+}$the upper directional Dini derivative is defined as follows:

$$
D^{+} V\left[x_{t}(0)\right] f\left(x_{t}\right)=\lim _{h \rightarrow 0^{+}} \sup \frac{V\left[x_{t}(0)+h f\left(x_{t}\right)\right]-V\left[x_{t}(0)\right]}{h} .
$$

A continuous function $\sigma: \mathbb{R}_{+} \rightarrow \mathbb{R}_{+}$belongs to the class $\mathcal{K}$ if it is strictly increasing and $\sigma(0)=0$; it belongs to the class $\mathcal{K}_{\infty}$ if it is also radially unbounded.

\subsection{Stability definitions}

Let $\Omega$ be an open subset of $C_{[-\tau, 0]}$ containing 0 .

Definition 1. Moulay et al. [12] The equilibrium $x=0$ of (1) is said to be

(a) stable if there is $\sigma \in \mathcal{K}$ such that for any $x_{0} \in \Omega,\left|x\left(t, x_{0}\right)\right| \leq \sigma\left(\left\|x_{0}\right\|\right)$ for all $t \geq 0$; 
(b) asymptotically stable if it is stable and $\lim _{t \rightarrow+\infty}\left|x\left(t, x_{0}\right)\right|=0$ for any $x_{0} \in \Omega$;

(c) finite-time stable if it is stable and for any $x_{0} \in \Omega$ there exists $0 \leq$ $T^{x_{0}}<+\infty$ such that $x\left(t, x_{0}\right)=0$ for all $t \geq T^{x_{0}}$. The functional $T_{0}\left(x_{0}\right)=$ $\inf \left\{T^{x_{0}} \geq 0: x\left(t, x_{0}\right)=0 \forall t \geq T^{x_{0}}\right\}$ is called the settling time of the system (1).

If $\Omega=C_{[-\tau, 0]}$, then the origin is called globally stable/asymptotically stable/finitetime stable.

For the forthcoming analysis we will need Lyapunov-Razumikhin theorem, which is given below (we have adapted to our case the formulation of $\mathrm{Gu}$ et al. [7], where time-dependent functional differential equations are considered).

Theorem 1. Gu et al. [7] Let $\alpha_{1}, \alpha_{2} \in \mathcal{K}$ and $\eta: \mathbb{R}_{+} \rightarrow \mathbb{R}_{+}$be a continuous nondecreasing function. If there exists a Lipschitz continuous function $V: \mathbb{R}^{n} \rightarrow$ $\mathbb{R}$ such that

$$
\alpha_{1}(|x|) \leq V(x) \leq \alpha_{2}(|x|) \quad \forall x \in \mathbb{R}^{n}
$$

and the derivative of $V$ along a solution $x(t)$ of (1) satisfies

$$
D^{+} V[x(t)] f\left(x_{t}\right) \leq-\eta(|x(t)|) \text { if } V[x(t+\theta)] \leq V[x(t)]
$$

for $\theta \in[-\tau, 0]$, then (1) is stable at the origin.

If, in addition, $\eta \in \mathcal{K}$ and there exists a continuous nondecreasing function $p(s)>s$ for $s>0$ such that the condition (2) is strengthened to

$$
D^{+} V[x(t)] f\left(x_{t}\right) \leq-\eta(|x(t)|) \text { if } V[x(t+\theta)] \leq p\{V[x(t)]\}
$$

for $\theta \in[-\tau, 0]$, then $(1)$ is asymptotically stable at the origin.

If in addition $\alpha_{1} \in \mathcal{K}_{\infty}$, then (1) is globally asymptotically stable.

\subsection{Sufficient conditions of finite-time stability in time-delay systems}

The following is stated in Yang and Wang [19] as Lemma 1 (an extension of the Lyapunov-Razumikhin method for analysis of finite-time stability in (1)):

"Consider the system (1) with $f(\phi)=F(\phi(0), \phi(-\tau)), \phi \in \Omega, F(0,0)=0$ and uniqueness of the solution in forward time. If there exist real numbers $\beta>1, k>0$, a Class- $\mathcal{K}$ function $\sigma$ and a differentiable Lyapunov function, $V(x)$, of the system (1) such that

$$
\begin{gathered}
\sigma(|x|) \leq V(x), \\
\dot{V} \leq-k V^{\beta^{-1}}(x), x \in \Omega
\end{gathered}
$$

hold along the trajectory of the system whenever $V[x(t+\theta)] \leq V[x(t)]$ for $\theta \in$ $[-\tau, 0]$, then the system (1) is finite-time stable. If $\Omega=\mathbb{R}^{n}$ and $\sigma$ is a Class- $\mathcal{K}_{\infty}$ function, then the origin is a globally finite-time stable equilibrium of the system 
(1). Furthermore, the settling time of the system (1) with respect to the initial condition $\phi \in C_{\delta}$ satisfies

$$
T_{0}(\phi) \leq \frac{\beta}{k(\beta-1)} V^{\frac{\beta-1}{\beta}}[\phi]
$$

for all $t \geq 0$."

For completeness we are going to give the "proof" of this lemma from Yang and Wang [19]:

"Since $V(x)$ is a Lyapunov function for the system (1), applying Razumikhin Theorem $\mathrm{Gu}$ et al. [7], it is easy to know that the system (1) is asymptotically stable under the conditions of the lemma. Next, we need to prove that $T_{0}(\phi)<$ $+\infty$. Based on Condition for $\dot{V}$, one can obtain $\int_{V(\phi)}^{0} \frac{d z}{z^{1 / \beta}} \leq-k \int_{0}^{t} d \tau$, from which it follows that $T_{0}(\phi) \leq \frac{\beta}{k(\beta-1)} V^{\frac{\beta-1}{\beta}}[\phi]$ for all $t \geq 0$. Thus, the proof is completed."

In Yang and Wang [18] the same conclusion is obtained for the time-varying system (1) using a similar argumentation.

\section{Comments on Yang and Wang [19]}

We claim that Lemma 1 in Yang and Wang [19] is incorrect as stated. Indeed, as we can conclude from the result of Theorem 1, Lemma 1 in Yang and Wang [19] is based on the condition (2), which allows only stability (non asymptotic) to be concluded. However, even if we would ask the inequality (3) to be satisfied whenever $V[\phi(\theta)] \leq p\{V[\phi(0)]\}$ for all $\theta \in[-\tau, 0]$, where $p: \mathbb{R}_{+} \rightarrow \mathbb{R}_{+}$is a continuous nondecreasing function such that $p(s)>s$ for all $s>0$, then only asymptotic stability can be proven following Theorem 1 . The claim about a finite-time convergence stays wrong since for a solution $x\left(t, x_{0}\right)$ with initial condition $x_{0} \in \Omega$ the Lyapunov-Razumikhin condition (3) defines the rate of convergence of $V$ only for the set of time instants

$$
\mathrm{T}_{x_{0}}^{+}=\left\{t \in \mathbb{R}_{+}: \sup _{\theta \in[-\tau, 0]} V\left[x\left(t+\theta, x_{0}\right)\right] \leq p\left\{V\left[x\left(t, x_{0}\right)\right]\right\}\right\}
$$

while for $t \in \mathrm{T}_{x_{0}}^{-}$with

$$
\mathrm{T}_{x_{0}}^{-}=\left\{t \in \mathbb{R}_{+}: \sup _{\theta \in[-\tau, 0]} V\left[x\left(t+\theta, x_{0}\right)\right]>p\left\{V\left[x\left(t, x_{0}\right)\right]\right\}\right\}
$$




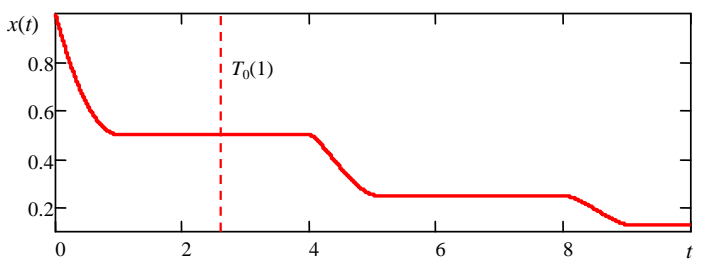

Figure 1: A trajectory of the counterexample (4)

there is no restriction on convergence rate of $V$. It is exactly the arguments used in the "proof" of Lemma 1 from Yang and Wang [19] given above: a finite-time rate of convergence is established for $t \in \mathrm{T}_{x_{0}}^{+}$only, which is clearly not sufficient since, for an illustration, if $V[x(t)]$ is strictly decreasing then $\mathrm{T}_{x_{0}}^{-}=[0,+\infty)$.

For another illustration consider the following counterexample:

$$
\dot{x}(t)=-|2 x(t)-x(t-\tau)|^{0.5} \operatorname{sign}[2 x(t)-x(t-\tau)] .
$$

Using $V(x)=0.5 x^{2}$ we obtain

$$
\dot{V} \leq-\sqrt{2-\sqrt{2}}|x|^{1.5}=-k V^{\beta^{-1}} \text { if } V[x(t-\tau)] \leq 2 V[x(t)]
$$

for $\beta=4 / 3$ and $k=\sqrt{2-\sqrt{2}} 2^{3 / 4}$. Thus, by Theorem 1 the system is globally asymptotically stable, and from Yang and Wang $[18,19]$ since all conditions of Lemma 1 Yang and Wang [19] are also satisfied, one would conclude that the system is finite-time stable. Consequently, for the initial conditions $x_{0} \in C_{[-\tau, 0]}$ such that $\left|x_{0}(0)\right| \leq 1$ the settling time function would possess the estimate:

$$
T_{0}\left(x_{0}\right) \leq 3
$$

Take $\tau>3$ and for any $\delta \in[-1,1] \backslash\{0\}$ select initial conditions

$$
x_{0}(s)=\left\{\begin{array}{ll}
2 \delta, & s \in[-\tau,-\tau+3] \\
\frac{\delta}{3-\tau} s+\delta, & s \in(-\tau+3,0]
\end{array} .\right.
$$

Obviously, $\dot{x}\left(t, x_{0}\right)=0$ and $x\left(t, x_{0}\right)=x_{0}(0)=\delta \neq 0$ for all $t \in\left[0, T_{0}\left(x_{0}\right)\right]$, therefore the given settling time estimate is invalid. In addition, the results of the system simulation for $x_{0}(s)=1$ for $s \in[-\tau, 0]$ are shown in Fig. 1 for $\tau=4$ and the simulation step $h=10^{-5}$. Clearly the system is not finite-time stable and the settling time estimate is wrong.

A peculiarity of the Lyapunov-Razumikhin conditions of stability is that they 
are delay-independent (see Theorem 1), thus the estimate on the settling time $T_{0}\left(x_{0}\right)$ obtained in Lemma 1 of Yang and Wang [19] is also delay independent. Consequently, it is possible to select delay value $\tau$ for a given initial conditions

$x_{0} \in C_{[-\tau, 0]}$ such that $T_{0}\left(x_{0}\right)<\tau$, as it has been performed in the counterexample above. Obviously, convergence to zero independently on the part of initial conditions $x_{0}(t)$ with $t \in\left[T_{0}\left(x_{0}\right)-\tau, 0\right]$ is possible only under special restrictions on $f$ in (1), which we are going to analyze in the next section.

\section{About necessary conditions for finite-time stability in time-delay systems}

Assume that the system (1) is finite-time stable for some $\tau>0$ and the settling-time functional is continuous and $T_{0}(0)=0$. Then there is $x_{0} \in C_{[-\tau, 0]}$ such that $T_{0}\left(x_{0}\right) \leq \tau$ and at the instant $T_{0}\left(x_{0}\right)$ the right-hand side of $(1)$ is still dependent on the initial conditions $x_{0}$. Thus, without additional assumptions on the right-hand side $f\left(x_{t}\right)$ and its dependence on $x_{t}(0)$, or without skipping the continuity requirement of $T_{0}\left(x_{0}\right)$, an existence of finite-time stability phenomenon for time-delay systems is questionable.

In the remainder of this section we will consider

$$
f(\phi)=F(\phi(0), \phi(-\tau))
$$

for all $\phi \in \Omega$, where $F: \mathbb{R}^{2 n} \rightarrow \mathbb{R}^{n}$ is a continuous function. Our conjecture is that the condition $F(0, z)=0$ for any $z \in \mathbb{R}^{n}$ is "necessary" for a finite-time convergence of (1) from $\Omega$ to the origin.

Remark 1. We would like to stress that requirement on continuity of $F$ is crucial for the consideration below. Indeed in discontinuous time-delay systems the finite-time stability may be easily observed without such a restriction on $F$. For example, it is a simple exercise to check that the system

$$
\dot{x}(t)=-(1+|x(t-\tau)|) \operatorname{sign}[x(t)]+x(t-\tau)
$$

is globally finite-time stable (take $V(x)=0.5 x^{2}, \dot{V} \leq-\sqrt{2 V}$ and apply a variant of Proposition 2). In this discontinuous case $0 \in F(0, z)$ for any $z \in \mathbb{R}^{n}$.

Note that by the definition of finite-time convergence $x\left(t, x_{0}\right)=0$ for all $t \geq T_{0}\left(x_{0}\right)$ and by the definition of $T_{0}\left(x_{0}\right)$ there is a non-empty set of time instants

$$
\mathcal{T}_{x_{0}}=\left\{t \in\left[T_{0}\left(x_{0}\right)-\tau, T_{0}\left(x_{0}\right)\right]: x\left(t, x_{0}\right) \neq 0\right\}
$$


Proposition 1. Let (1) be finite-time convergent in $\Omega$, then

$$
\forall t \in \mathcal{T}_{x_{0}}: F\left[0, x\left(t, x_{0}\right)\right]=0
$$

for any $x_{0} \in \Omega$.

Proof. Take $x_{0} \in \Omega$ and the corresponding settling time $T_{0}\left(x_{0}\right)$. Assume that the necessary condition (5) is not satisfied, its negation implies that for some $x_{0} \in \Omega$ there exists $t^{\prime} \in \mathcal{T}_{x_{0}}$ such that $F\left(0, x\left(t^{\prime}, x_{0}\right)\right) \neq 0$ (the measure of $\mathcal{T}_{x_{0}}$ is not zero since $x(t)$ and $F$ both are continuous), then $\dot{x}\left(t^{\prime}+\tau\right) \neq 0$ and $x\left(t, x_{0}\right) \neq 0$ for some $t \geq T_{0}\left(x_{0}\right)$ that is a contradiction.

A simple, but not equivalent, way to check this condition in practice is to verify that

$$
F(0, z)=0
$$

for any $z \in \mathbb{R}^{n}$. In Kolmanovskii and Myshkis [10], a similar "necessary" condition has been indicated for the El'sgol'ts approach El'sgol'ts and Norkin [5]. Indeed, consider the case $n=1$, take a Lyapunov function $V(x)=0.5 x^{2}$, which is a reasonable choice for the scalar case. We have $\dot{V}(t)=x(t) F[x(t), x(t-\tau)]$, if the inequality $\dot{V}(t) \leq 0$ is satisfied around the origin, then we necessarily obtain (6).

In Yang and Wang $[18,19]$ the restriction $F(0,0)=0$ has been imposed that is not sufficient.

\section{Discussion}

Finite-time stability can be presented in time-delay systems, but only under rather strong restrictions on the right-hand side of the system. The most general sufficient conditions are given in Moulay et al. [12]:

Proposition 2. Moulay et al. [12] Let system (1) have unique solutions in forward time. If there exist a continuous functional $V: \Omega \rightarrow \mathbb{R}_{+}, \epsilon>0$ and two functions $\alpha, r$ of class $\mathcal{K}$ such that $\dot{z}=-r(z)$ has a flow, $\int_{0}^{\epsilon} \frac{d z}{r(z)}<+\infty$ and for all $\phi \in \Omega$

$$
\alpha(|\phi(0)|) \leq V(\phi), D^{+} V(\phi) \leq-r[V(\phi)],
$$

then the system (1) is finite-time stable with a settling time functional $T_{0}(\phi)$ satisfying the inequality:

$$
T_{0}(\phi) \leq \int_{0}^{V(\phi)} \frac{d z}{r(z)} .
$$

The following example has been given in Moulay et al. [12] for any $0<\alpha<1$ :

$$
\dot{x}(t)=-|x(t)|^{\alpha} \operatorname{sign}[x(t)]\left\{1+x(t-\tau)^{2}\right\} .
$$


Using the Lyapunov functional $V(\phi)=0.5 \phi(0)^{2}$ with $\dot{V}(\phi) \leq-2^{\frac{1+\alpha}{2}} V^{\frac{1+\alpha}{2}}(\phi)$ the finite-time stability has been established. Note that for this example the "necessary" condition (6) is satisfied.

The system (7) is an example, where the El'sgol'ts' arguments can be applied $\left(V(\phi)=0.5 \phi(0)^{2}\right.$ is in fact a Lyapunov function). Note that the result of Lemma 1 in Yang and Wang [19] without the Razumikhin condition ("whenever...") is correct and in this case it is also a special case of Proposition 2. In such a reformulation the result extends El'sgol'ts and Norkin [5] to finite-time stability, as well as the finite-time stability results of Roxin [17] to time-delay systems.

\section{Acknowledgment}

This work was partially supported by the Government of Russian Federation (Grant 074-U01), the Ministry of Education and Science of Russian Federation (Project 14.Z50.31.0031) and by Israel Science Foundation (grant No 754/10).

[1] Andrieu, V., Praly, L., Astolfi, A., 2008. Homogeneous approximation, recursive observer design, and output feedback. SIAM J. Control Optimization 47 (4), 1814-1850.

[2] Bhat, S., Bernstein, D., 2005. Geometric homogeneity with applications to finite-time stability. Mathematics of Control, Signals and Systems 17, 101-127.

[3] Chiasson, J., Loiseau, J. (Eds.), 2007. Applications of Time Delay Systems. Vol. 352 of Lecture Notes in Control and Information Sciences. Springer.

[4] Dorato, P., 2006. An overview of finite-time stability. In: Menini, L., Zaccarian, L., Abdallah, C. T. (Eds.), Current Trends in Nonlinear Systems and Control. Systems \& Control: Foundations \& Applications. Birkhäuser, Boston, pp. 185-194.

[5] El'sgol'ts, L., Norkin, S., 1973. Introduction to the theory and application of differential equations with deviating arguments. Vol. 105 of Mathematics in Science and Engineering. Academic Press, New York.

[6] Erneux, T., 2009. Applied Delay Differential Equations. Springer, NY.

[7] Gu, K., Kharitonov, K., Chen, J., 2003. Stability of Time-Delay Systems. Control Engineering. Birkhäuser, Boston. 
[8] Hale, J., 1977. Theory of Functional Differential Equations. SpringerVerlag.

[9] Karafyllis, I., 2006. Finite-time global stabilization by means of timevarying distributed delay feedback. SIAM J. Control Optim. 45, 320-342.

[10] Kolmanovskii, V., Myshkis, A., 1999. Introduction to the Theory and Applications of Functional Differential Equations. Vol. 463 of Mathematics and Its Applications. Springer.

[11] Kolmanovsky, V., Nosov, V., 1986. Stability of functional differential equations. CA: Academic, San Diego.

[12] Moulay, E., Dambrine, M., Yeganefar, N., Perruquetti, W., 2008. Finitetime stability and stabilization of time-delay systems. Systems Control Lett. $57,561-566$.

[13] Moulay, E., Perruquetti, W., 2006. Finite time stability and stabilization of a class of continuous systems. J. Mathematical Analysis Applications 323 (2), 1430-1443.

[14] Nersesov, S., Haddad, W., Hui, Q., 2008. Finite-time stabilization of nonlinear dynamical systems via control vector lyapunov functions. J. Franklin Inst. 345, 819-837.

[15] Polyakov, A., 2012. Nonlinear feedback design for fixed-time stabilization of linear control systems. Automatic Control, IEEE Transactions on 57 (8), $2106-2110$.

[16] Richard, J.-P., 2003. Time-delay systems: an overview of some recent advances and open problems. Automatica 39, 1667-1694.

[17] Roxin, E., 1966. On finite stability in control systems. Rendiconti del Circolo Matematico di Palermo 15, 273-283.

[18] Yang, R., Wang, Y., 2012. Finite-time stability and stabilization of a class of nonlinear time-delay systems. SIAM J. Control Optim. 50 (5), 31133131.

[19] Yang, R., Wang, Y., 2013. Finite-time stability analysis and $H_{\infty}$ control for a class of nonlinear time-delay Hamiltonian systems. Automatica 49, 390-401. 